\title{
Chemokine-Induced Secretion of Gelatinase B in Primary Human Monocytes
}

\author{
Christiane M. Klier ${ }^{1}$, Edward L. Nelson², \\ Clemens D. Cohen ${ }^{1}$, Richard Horuk ${ }^{3}$, Detlef \\ Schlöndorff' ${ }^{1}$ and Peter J. Nelson ${ }^{1, *}$ \\ ${ }^{1}$ Medizinische Poliklinik, Ludwig-Maximilians- \\ Universität, D-80336 Munich, Germany \\ ${ }^{2}$ Department of Medicine, Division of Hematology and \\ Oncology, University of California, Irvine, CA 92697 , \\ USA \\ ${ }^{3}$ Berlex Biosciences, Richmond, CA 94305, USA \\ ${ }^{*}$ Corresponding author
}

Chemokines help control normal leukocyte trafficking as well as their infiltration into tissues during acute and chronic inflammation. Matrix metalloproteinases (MMPs) help support the extravasation and infiltration of leukocytes through limited proteolysis of basement membranes and matrix material. The effect of the chemokines RANTES/CCL5, MCP-1/CCL and SDF-1/CXCL12 on secretion of the matrix metalloproteinase B and its endogenous inhibitor TIMP-1 was studied. RANTES/CCL5 and SDF-1/CXCL12 were found to induce MMP-9 secretion in primary human monocytes while TIMP-1 secretion was not affected. RANTES/CCL5 effects were mediated through CCR1 because the CCR1 antagonist BX471 was found to effectively block RANTES/CCL5-induced MMP-9 secretion.

Key words: BX471/CCR1/CXCR4 / MMP-9/RANTES / TIMP-1.

Chemokines are a large family of chemotactic cytokines that control diverse biological actions including cellular trafficking, hematopoiesis and angiogenesis (Nelson and Krensky, 2001). Select chemokines control acute and chronic inflammatory processes such as those found in rheumatoid arthritis (DeVries et al., 1999), allograft transplant rejection (Grone et al., 1999; Horuk et al., 2001; Nelson and Krensky, 2001) and multiple sclerosis (Ransohoff, 1999). In addition, chemokines and their receptors have been implicated in tumor growth and metastasis (Muller et al., 2001).

The matrix metalloproteinases (MMPs) play critical roles in health and disease and are represented by at least 20 extracellular matrix degrading metallo endopetidases (Nagase, 1997; Nagase and Woessner, 1999).
These enzymes are active during tissue development and differentiation, cellular infiltration and wound healing (Nagase, 1997; Nagase and Woessner, 1999). MMPs have also been implicated in chronic disease processes such as multiple sclerosis and rheumatoid arthritis, as well as in tumor progression and metastasis (Stetler-Stevenson et al., 1993a; Murphy and Gavrilovic, 1999).

Based upon their substrate specificity and cellular location, the MMPs have been ascribed to select subgroups. These groups include the collagenases, stromelysins, gelatinases and membrane-bound matrix metalloproteinases (MT-MMP) (Nagase, 1997; Nagase and Woessner, 1999). The endogenous tissue inhibitors of matrix metalloproteinases, or TIMPs, regulate the enzymatic activity of MMPs. To date, four TIMP proteins (TIMP-1, 2, 3, and 4) have been described (Nagase and Woessner, 1999; Brew et al., 2000). A balance between MMP and TIMP protein interactions is thought to help control the functional activity of MMPs in vivo (Murphy and Gavrilovic, 1999; Nagase and Woessner, 1999).

Chemokines help direct the influx and migration of select immune effector cells, while MMPs are involved in the breakdown of the extracellular matrix (Stuve et al., 1996; Ozenci et al., 1999; Nelson and Krensky, 2001). Recent observations have highlighted the biological linkage between these two families of proteins (Nelson and Krensky, 2001). For example, the functional activity of chemokines can be modified through post-translational proteolytic processing. Cleavage of MCP-3/CCL7 by the matrix metalloproteinase gelatinase A (MMP2) generates a product that binds to the chemokine receptors CCR1, CCR2, and CCR3 and acts as a potent antagonist that suppresses inflammation, thus demonstrating that matrix metalloproteinases can act both as effectors and regulators of the inflammatory response (McQuibban et al., 2000).

Since chemokines direct leukocyte migration it has been proposed that they may also control the production of MMPs by migrating monocytes. MMPs in general, and specifically the gelatinases A (MMP-2) and B (MMP-9), are thought to be of pivotal importance in leukocyte extravasation (Nagase and Woessner, 1999). Monocytes are equipped with a series of chemokine receptors that control their normal trafficking as well as migration during inflammation. We hypothesized that chemokines that elicit monocyte migration may also control production of the MMPs required for extravasation. This question was pursued from two biological perspectives. The first fo- 
cused on chemokines/chemokine receptors that control normal monocyte trafficking - independent of inflammation. The second focused on chemokines and receptors linked to monocyte infiltration during acute and chronic inflammatory processes.

Freshly isolated peripheral blood monocytes were first characterized for chemokine receptor, gelatinase $B$ (MMP-9), and TIMP-1 expression. The cells were then stimulated with various concentrations of appropriate chemokine ligands. Consistent with earlier reports, freshly isolated human peripheral blood monocytes were found to express the chemokine receptors CCR1, CCR2 and CXCR4 as assessed by fluorescence-assisted cell sorting (FACS; Figure 1A, C and D) and PCR analysis (Mack et al., 2000; Weber et al., 2001). CCR1 and CCR2 are proinflammatory chemokine receptors, while the receptor CXCR4 is involved in leukocyte homeostasis (Nelson and Krensky, 2001). The receptor CCR5 was found to be upregulated on the monocytes but only after incubation/activation on plastic for 24 hours (data not shown). The primary monocytes also strongly expressed MMP-9 and TIMP-1 on their surface (Figure 1E and F).

The monocytes (200 000 cells/well) were treated with increasing concentrations of chemokines that target the various chemokine receptors: RANTES/CCL5; CCR1, MCP-1/CCL2; CCR2, and SDF-1/CXCL12; CXCR4. The resultant conditioned culture medium was analyzed by gelatin-zymography (Figure 2). Stimulation with TNF- $\alpha$ (10 ng/ml) served as a positive control for induced MMP production (Zhang et al., 1998). Analysis revealed that the chemokines RANTES/CCL5 and SDF-1/CXCL12 both induced a release of pro-MMP-9 (Figure 2). A variable response was also seen with MCP-1. Maximum MMP-9 protein secretion was observed after 24 to 36 hours of chemokine incubation as tested over a range of $48 \mathrm{~h}$ (data not shown). No secretion of MMP-2 (gelatinase A) protein was seen.

To quantify the MMP-9 protein secreted into the culture medium, an MMP-9-specific substrate assay was employed. MMP-9 protein could be assessed by the substrate assay only after activation of the conditioned medium with $p$-aminophenyl mercuric acetate (APMA), demonstrating the secretion of pro-MMP-9 by the monocytes. Again, RANTES/CCL5 and SDF-1/CXCL12 were found to induce pro-MMP-9 production (Figure $3 A$ ). Stimulation with $200 \mathrm{ng} / \mathrm{ml}$ RANTES/CCL5 or SDF$1 / C X C L 12$ represented the maximal level of stimulation seen and resulted in a 5- and 7-fold increased secretion of pro-MMP-9 protein, respectively (Figure 3A).

The MCP-1-induced expression of MMP-9 by monocytes has been previously described (Vos et al., 2000). In the experiments detailed here, a slight increase of MMP9 secretion was sometimes seen following MCP-1 stimulation. However, these results were not consistent. Essentially no increase in pro-MMP-9 secretion was seen in three separate experiments, an increase (up to 3.5-fold as determined by MMP-9 substrate assay) was found in two experiments. An increase of MMP-9 after SDF-1
A

B
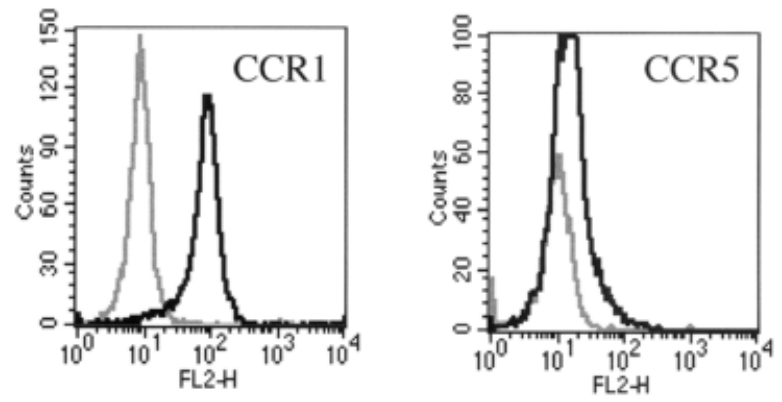

$\mathrm{C}$

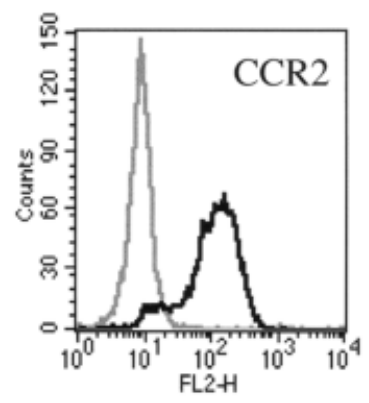

D

E

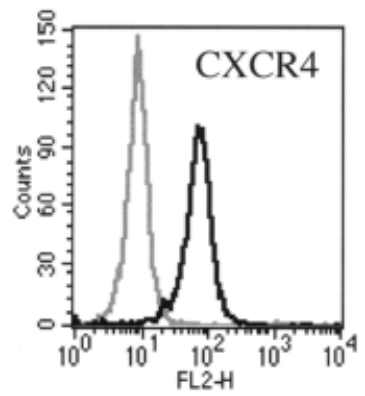

F

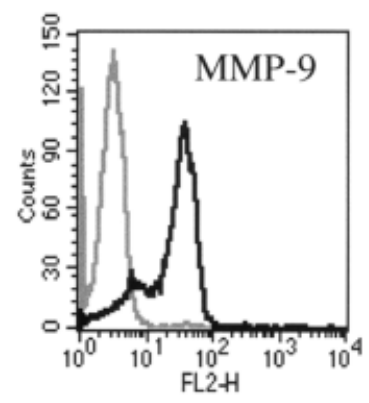

Fig. 1 FACS Analysis of Cell Surface Chemokine Receptor Expression on Monocytes.

Flow cytometric analysis was performed as described (Mack et al., 2000; Weber et al., 2001). Freshly isolated monocytes were stained with antibodies directed against a series of chemokine receptors, MMP-9 and TIMP-1. (A) CCR1, MAB145 (R\&D Systems, Wiesbaden, Germany); (B) CCR5, mAB clone MC-1 kindly provided by Dr. M. Mack (Ludwig-Maximilans-University, Munich, Germany); (C) CCR2, MAB 150 (R\&D Systems); (D) CXCR4, MAB 170 clone \# 12G5 (R\&D Systems); (E) MMP-9, IM09L (Calbiochem-Novabiochem $\mathrm{GmbH}$, Bad Soden, Germany); and (F) TIMP-1, IM32L (Calbiochem-Novabiochem). Data are expressed as overlay histogram analysis of specific anti-CCR or CXCR signal (bold line) and isotype control (thin line).

stimulation of $\mathrm{CD} 34^{+}$cells has recently been described (Janowska-Wieczorek et al., 2000).

The natural inhibitor for MMP-9 is TIMP-1 (Nagase and Woessner, 1999). Analysis of conditioned medium using a TIMP-1-specific ELISA (Amersham Pharmacia, Freiburg, Germany) showed no significant effect on TIMP-1 secretion following chemokine stimulation (Figure 3B). FACS analysis of the monocytes also showed no increase in surface expression of TIMP-1 following 


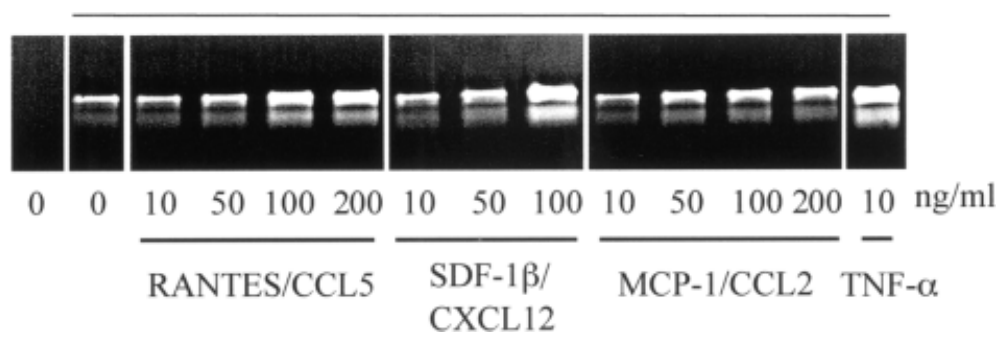

Fig. 2 Chemokine-Induced Secretion of proMMP-9 in Primary Monocytes.

Gelatin-zymogram of RANTES/CCL5-induced secretion of pro-MMP-9. Monocytes were isolated from EDTA-blood of healthy volunteers as described (Weber et al., 2001) and cultured in Macrophage SFM (Gibco-BRL Life Technologies, Karlsruhe, Germany) at a density of $1.5-2.0 \times 10^{6} \mathrm{cells} / \mathrm{ml}$ in 96-well plates (Costar Corning, Cambridge MA, USA). Cells were stimulated as shown with RANTES/CCL5, MCP-1/CCL2, SDF-1/CXCL12 or TNF- $\alpha$ (all from TEBU, Frankfurt, Germany) for $24 \mathrm{~h}$. Conditioned media were stored at $-20^{\circ} \mathrm{C}$.

The concentrations of chemokines used (between 10 and $200 \mathrm{ng} / \mathrm{ml}$ ) represent the general range of protein concentrations used to establish optimal chemotactic parameters for chemokine induced migration of primary monocytes (Horuk and Ng, 2000; Weber et al., 2001). The media supernatants from monocytes were mixed with $2 \times$ loading buffer (Invitrogen, Groningen, Netherlands). Proteins were separated on a precast $10 \%$ Tris-glycine gel containing $0.1 \%$ gelatin (Invitrogen). Following electrophoreses, renaturing and developing was performed according to manufacturer's recommendation. Gel development was stopped after 3 to 5 hours of incubation at $37{ }^{\circ} \mathrm{C}$. Equivalent results were obtained in three independent experiments.
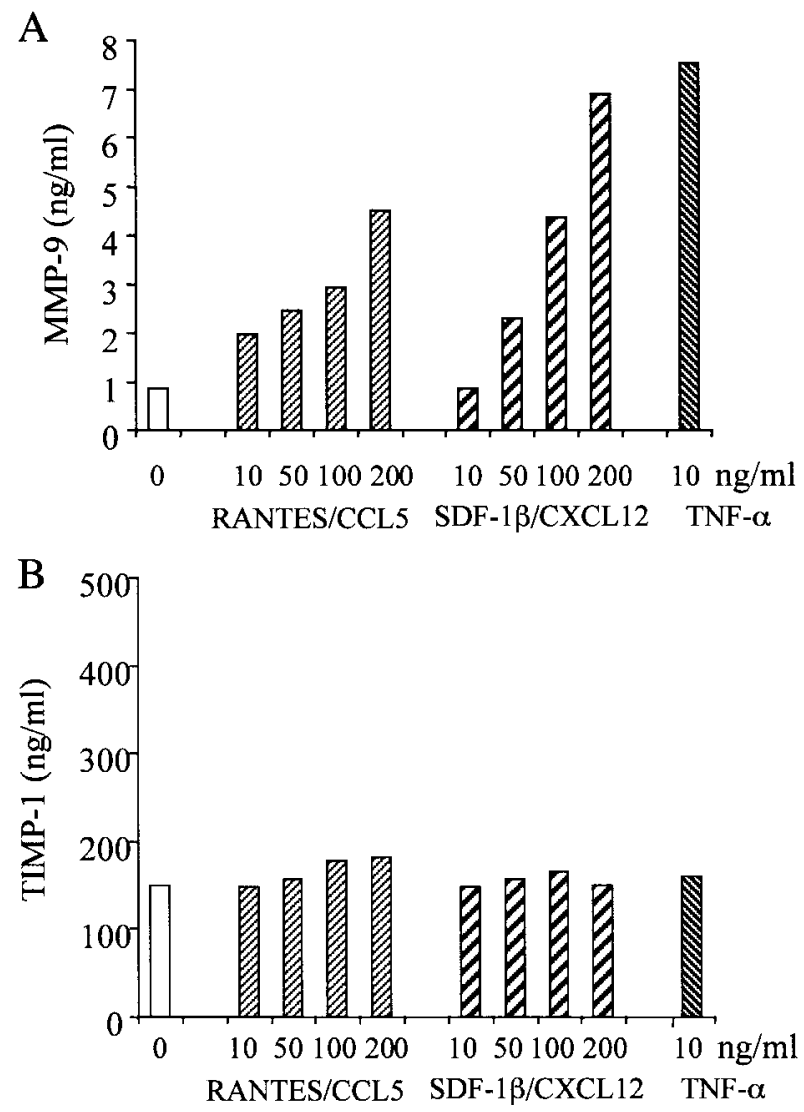

chemokine stimulation (data not shown). Analysis of MMP-9 mRNA expression after stimulation with RANTES/CCL 5 by real time RT-PCR (TaqMan, Perkin Elmer-ABI, Weiterstadt, Germany) revealed a 2.4-fold increase in steady-state mRNA expression after stimulation with RANTES/CCL5, demonstrating control at the mRNA level (data not shown).

Macrophages have been reported to secrete MMP-1
Fig. 3 Quantification of Chemokine-Induced Secretion of MMP-9 and TIMP-1 Proteins.

Cells were cultured in Macrophage SFM at a density of 1.5-2.0 $\times 10^{6} \mathrm{cells} / \mathrm{ml}$ for $24 \mathrm{~h}$. Conditioned media were harvested and stored at $-20^{\circ} \mathrm{C}$.

(A) Quantitation of chemokine-induced pro-MMP-9 in the conditioned medium was analyzed by an MMP-9 substrate assay \#RPN 2634 (Amersham Pharmacia) according to manufacturers recommendations for the detection of $0.125-4.0 \mathrm{ng} / \mathrm{ml}$ MMP-9 protein. The results presented are an average from two values. Essentially equivalent results were obtained in three independent experiments.

(B) TIMP-1 protein was measured using a specific ELISA \#RPN 6211 (Amersham Pharmacia) following manufacturers recommendations. Absorbance at 450nm was read via an Emax Elisa Reader (Molecular Devices, Ismaning/Munich, Germany) and analyzed with Softmax ${ }^{\circledR}$ pro analysis software (Molecular Devices). The values presented are an average of parallel measurements and represent the results seen in three independant experiments.

(Corcoran et al., 1995) and MMP-3 (Shapiro et al., 1990). Analysis of conditioned medium by MMP-1 or MMP-3 Biotrak ELISA (Amersham Pharmacia) could not demonstrate RANTES-induced secretion of MMP-1 nor of MMP-3 in the primary monocytes (data not shown). However, 'activated' (24 hours on plastic) monocytes did show low levels of MMP-3 by ELISA (data not shown). This is in contrast to recently published findings (Borzi et al., 2000) describing RANTES/CCL5-induced secretion of MMP-3 at a level above $1000 \mathrm{ng} / \mathrm{ml}$ in normal and osteoarthritic chondrocytes.

Primary monocytes can in theory bind RANTES/CCL5 through at least two chemokine receptors, CCR1 and CCR5 (Nelson and Krensky, 2001). Freshly isolated monocytes do not express CCR3, an additional RANTES/CCL5 receptor, as assessed by FACS analysis and RT-PCR (Weber et al., 2001) but do strongly express CCR1 (Figure 1). CCR5 is not expressed by freshly isolat- 
ed monocytes (Figure 1), but is upregulated upon activation (Mack et al., 2000; Weber et al., 2001).

The chemokine receptor CCR1 has been identified as a potential therapeutic target for control of acute and chronic inflammation through a selective blockade of leukocyte recruitment (Grone et al., 1999; Horuk et al., 2001; Nelson and Krensky, 2001). To demonstrate the role of CCR1 in the RANTES/CCL5-induced upregulation and secretion of MMP-9 by monocytes, a biologically active non-peptide antagonist (BX 471) directed against CCR1 was employed (Horuk et al., 2001).

Following preincubation of monocytes with BX 471 (10 $\mu \mathrm{M})$, the cells were stimulated with RANTES/CCL5 for 24 h. Secretion of pro-MMP-9 was assessed both by gelatinzymography (Figure 4A) and MMP-9 substrate assay (Figure $4 \mathrm{~B})$. TNF- $\alpha$ (10 $\mathrm{ng} / \mathrm{ml})$ stimulation served as positive control. In comparison to vehicle control (DMSO), the CCR1 antagonist caused a significant reduction of MMP9 release into the culture medium (Figure 4A). Release of TNF- $\alpha$ induced MMP-9 was not affected by BX 471
(Figure 4A). The essentially complete blockage of RANTES/CCL5-induced secretion of pro-MMP-9 by BX 471 clearly demonstrates the importance of CCR 1 in mediating the RANTES/CCL5-induced secretion of MMP-9. The slight reduction in constitutive MMP-9 secretion seen in the absence of RANTES/CCL5 stimulation may be due to the low constitutive expression of RANTES/CCL5 seen in freshly isolated human monocytes (data not shown).

Analysis of the specialized roles of apparently redundant chemokine receptors has demonstrated the central importance of CCR1 in the direct recruitment of monocytes from the peripheral circulation (Weber et al., 2001). During inflammation chemokines such as RANTES/CCL5 may help induce the production of MMP-9 by infiltrating CCR1-expressing monocytes. Thus, a selective blockade of CCR1 may help control tissue damage in part, through suppression of MMP-9 production. To this end, CCR1 antagonists have been shown to dramatically reduce the tissue damage associated with allograft transplant rejection and multiple sclerosis (Grone et al., 1999;

A
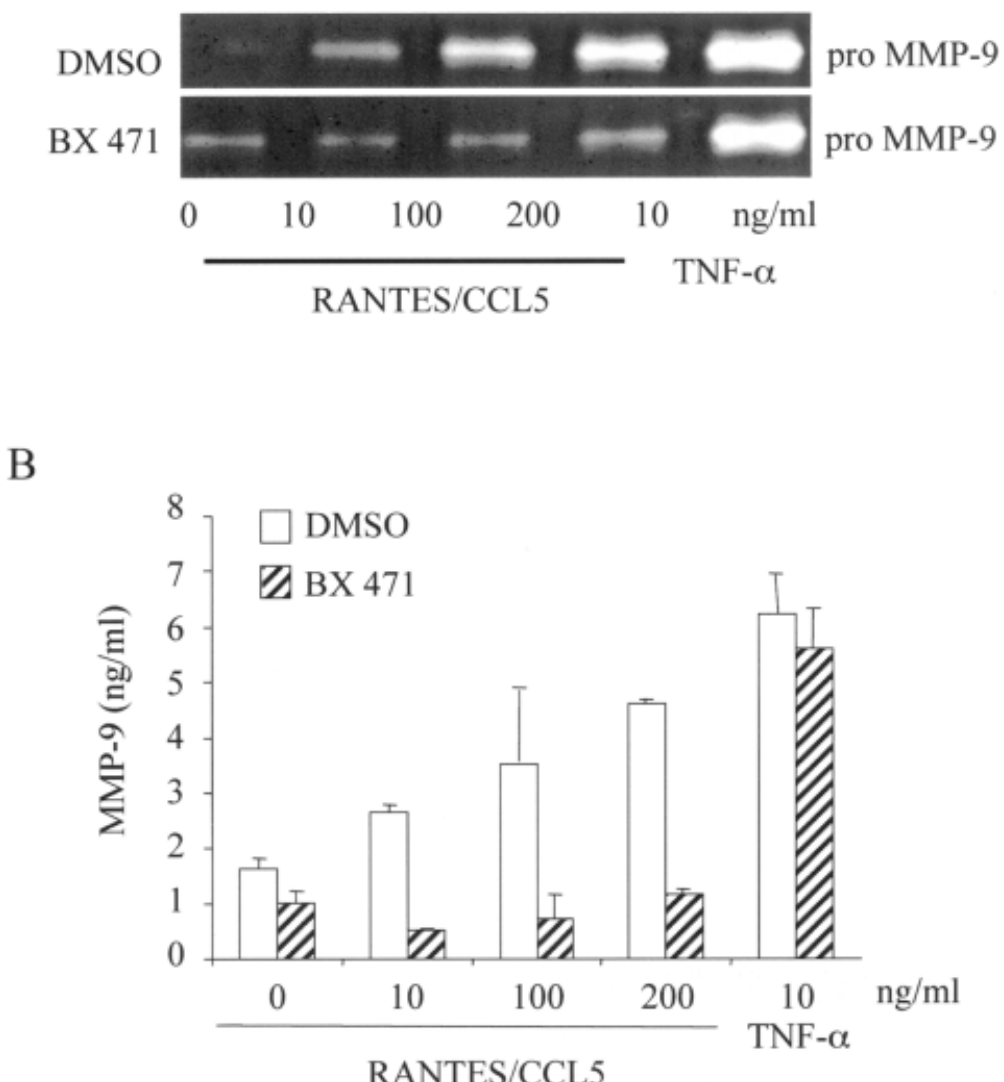

Fig. 4 Effect of the Anti-CCR1 Antagonist BX 471 on RANTES-Induced Secretion of MMP-9.

Freshly isolated monocytes were preincubated with BX $471(10 \mu \mathrm{M})$ for $10 \mathrm{~min}$ followed by stimulation with increasing concentrations of RANTES/CCL5 (see Figure) or TNF- $\alpha(10 \mathrm{ng} / \mathrm{ml})$ in Macrophage SFM at a density of $1.5-2.0 \times 10^{6} \mathrm{cells} / \mathrm{ml}$ for $24 \mathrm{~h}$. Conditioned medium was harvested and stored at $-20^{\circ} \mathrm{C}$ for further analysis.

(A) Gelatine zymogram of conditioned medium.

(B) Quantification of MMP-9 concentration by MMP-9 substrate assay. Results presented are the average of three determinations \pm the standard error of the mean. Results were confirmed in three independant experiments. 
Horuk and Ng, 2000; Horuk et al., 2001; Nelson and Krensky, 2001).

Tumor expression of RANTES/CCL5 has been linked to 'aggressiveness' of breast carcinoma (Luboshits et al., 1999). Increased production of RANTES/CCL5 by the tumor correlates with a more advanced stage of the disease. Breast cancer staging has also been associated with increased mononuclear cell infiltration (UrdialesViedma et al., 1986). Furthermore, it is recognized that MMP activity is involved in the control of tumor growth and metastasis (Stetler-Stevenson et al., 1993b; Nagase and Woessner, 1999). Based upon the results presented here, one could speculate that RANTES-induced attraction and activation of monocytes may lead to MMP-9 mediated degradation of the extracellular matrix surrounding the tumor tissue, thereby promoting tumor proliferation, and potentially, tumor escape.

We have further shown that the homeostatic chemokine SDF-1/CXCL12, acting through CXCR4, can moderate MMP-9 production by primary monocytes, a mechanism that may be important to normal monocyte trafficking. Interestingly, a recent report has demonstrated the importance of CXCR4 expression by breast cancer cells in promoting tumor metastasis to bone marrow. If the metastatic tumor cell behaves in a manner analogous to that seen in monocytes, then SDF-1 may also directly induce MMP production by these cells, thus facilitating tumor cell egress and invasion of other SDF-1 expressing tissues.

The results presented here suggest that chemokine receptor blockade may limit tissue damage by suppressing MMP production, and may in addition help limit tumor progression.

\section{Acknowledgements}

The authors would like to thank Anke Mojaat for technical assistance. Support was provided by a grant to P.J.N. from the Deutsche Forschungsgemeinschaft, Sonderforschungsbereich 469.

\section{References}

Borzi, R. M., Mazzetti, I., Cattini, L., Uguccioni, M., Baggiolini, M., and Facchini, A. (2000). Human chondrocytes express functional chemokine receptors and release matrix-degrading enzymes in response to $\mathrm{C}-\mathrm{X}-\mathrm{C}$ and $\mathrm{C}-\mathrm{C}$ chemokines. Arthritis Rheum. 43, 1734-1741.

Brew, K., Dinakarpandian, D., and Nagase, H. (2000). Tissue inhibitors of metalloproteinases: evolution, structure and function. Biochim. Biophys. Acta 1477, 267-283.

Corcoran, M. L., Kibbey, M. C., Kleinman, H. K., and Wahl, L. M. (1995). Laminin SIKVAV peptide induction of monocyte/macrophage prostaglandin E2 and matrix metalloproteinases. J. Biol. Chem. 270, 10365-10368.

DeVries, M. E., Ran, L., and Kelvin, D. J. (1999). On the edge: the physiological and pathophysiological role of chemokines during inflammatory and immunological responses. Semin. Immunol. 11, 95-104.
Grone, H. J., Weber, C., Weber, K. S., Grone, E. F., Rabelink, T., Klier, C. M., Wells, T. N., Proudfood, A. E., Schlondorff, D., and Nelson, P. J. (1999). Met-RANTES reduces vascular and tubular damage during acute renal transplant rejection: blocking monocyte arrest and recruitment. FASEB J. 13, 1371-1383.

Horuk, R., Clayberger, C., Krensky, A. M., Wang, Z., Grone, H. J., Weber, C., Weber, K. S., Nelson, P. J., May, K., Rosser, M. et al. (2001). A non-peptide functional antagonist of the CCR1 chemokine receptor is effective in rat heart transplant rejection. J. Biol. Chem. 276, 4199-4204.

Horuk, R., and Ng, H. P. (2000). Chemokine receptor antagonists. Med. Res. Rev. 20, 155-168.

Janowska-Wieczorek, A., Marquez, L. A., Dobrowsky, A., Ratajczak, M. Z., and Cabuhat, M. L. (2000). Differential MMP and TIMP production by human marrow and peripheral blood $\mathrm{CD} 34^{+}$cells in response to chemokines. Exp. Hematol. 28, $1274-1285$.

Luboshits, G., Shina, S., Kaplan, O., Engelberg, S., Nass, D., Lifshitz-Mercer, B., Chaitchik, S., Keydar, I., and Ben-Baruch, A. (1999). Elevated expression of the CC chemokine regulated on activation, normal T cell expressed and secreted (RANTES) in advanced breast carcinoma. Cancer Res. 59, 4681-4687.

Mack, M., Kleinschmidt, A., Bruhl, H., Klier, C., Nelson, P. J., Cihak, J., Plachy, J., Stangassinger, M., Erfle, V., and Schlondorff, D. (2000). Transfer of the chemokine receptor CCR5 between cells by membrane-derived microparticles: a mechanism for cellular human immunodeficiency virus 1 infection. Nature Med. 6, 769-775.

McQuibban, G. A., Gong, J. H., Tam, E. M., McCulloch, C. A., Clark-Lewis, I., and Overall, C. M. (2000). Inflammation dampened by gelatinase A cleavage of monocyte chemoattractant protein-3. Science 289, 1202-1206.

Muller, A., Homey, B., Soto, H., Ge, N., Catron, D., Buchanan, M. E., McClanahan, T., Murphy, E., Yuan, W., Wagner, S. N. et al. (2001). Involvement of chemokine receptors in breast cancer metastasis. Nature 410, 50-56.

Murphy, G., and Gavrilovic, J. (1999). Proteolysis and cell migration: creating a path? Curr. Opin. Cell Biol. 11, 614-621.

Nagase, H. (1997). Activation mechanisms of matrix metalloproteinases. Biol. Chem. 378, 151-160.

Nagase, H., and Woessner, J. F. (1999). Matrix metalloproteinases. J. Biol. Chem. 274, 21491-21494.

Nelson, P. J., and Krensky, A. M. (2001). Chemokines and chemokine receptors in allograft rejection. Immunity 14, $377-386$.

Ozenci, V., Rinaldi, L., Teleshova, N., Matusevicius, D., Kivisakk, P., Kouwenhoven, M., and Link, H. (1999). Metalloproteinases and their tissue inhibitors in multiple sclerosis. J. Autoimmun. 12, $297-303$.

Ransohoff, R. M. (1999). Mechanisms of inflammation in MS tissue: adhesion molecules and chemokines. J. Neuroimmunol. 98, 57-68.

Shapiro, S. D., Campbell, E. J., Kobayashi, D. K. and Welgus HG. (1990). Immune modulation of metalloproteinase production in human macrophages. Selective pretranslational suppression of interstitial collagenase and stromelysin biosynthesis by interferon- $\gamma$. J. Clin. Invest. 86, 1204-1210.

Stetler-Stevenson, W. G., Liotta, L. A., and Kleiner, D. E. (1993a). Extracellular matrix 6: role of matrix metalloproteinases in tumor invasion and metastasis. FASEB J. 7, 1434-1441.

Stetler-Stevenson, W. G., Aznavoorian, S., and Liotta, L. A. (1993b). Tumor cell interactions with the extracellular matrix during invasion and metastasis. Annu. Rev. Cell. Biol. 9, $541-573$.

Stuve, O., Dooley, N. P., Uhm, J. H., Antel, J. P., Francis, G. S., Williams, G., and Yong, V. W. (1996). Interferon $\beta$-1b decreas- 
es the migration of $\mathrm{T}$ lymphocytes in vitro: effects on matrix metalloproteinase-9. Ann. Neurol. 40, 853-863.

Urdiales-Viedma, M., Nogales-Fernandez, F., Martos-Padilla, S., and Sanchez-Cantalejo, E. (1986). Correlation of histologic grade and lymph node status with some histopathologic discriminants in breast cancer. Tumori 72, 43-51.

Vos, C. M., Gartner, S., Ransohoff, R. M., McArthur, J. C., Wahl, L., Sjulson, L., Hunter, E., and Conant, K. (2000). Matrix metalloprotease- 9 release from monocytes increases as a function of differentiation: implications for neuroinflammation and neurodegeneration. J. Neuroimmunol. 109, 221-227.
Weber, C., Weber, K. S., Klier, C., Gu, S., Wank, R., Horuk, R., and Nelson, P. J. (2001). Specialized roles of the chemokine receptors CCR 1 and CCR5 in the recruitment of monocytes and $\mathrm{T}(\mathrm{H}) 1$-like/CD45RO+ $\mathrm{T}$ cells. Blood 97, 1144-1146.

Zhang, Y., McCluskey, K., Fujii, K., and Wahl, L. M. (1998). Differential regulation of monocyte matrix metalloproteinase and TIMP-1 production by TNF- $\alpha$, granulocyte-macrophage CSF, and IL-1 $\beta$ through prostaglandin-dependent and -independent mechanisms. J. Immunol. 161, 3071-3076.

Received April 20, 2001; accepted June 15, 2001 